NASA-CR-205002

\title{
Modeling Piezoelectric Stack Actuators for Control of Micromanipulation
}

\section{Michael Goldfarb and Nikola Celanovic$$
0 \geqslant 6764
$$

A nonlinear lumped-parameter model of a piezoelectric stack actuator has been developed to describe actuator behavior for purposes of control system analysis and design, and, in particular, for microrobotic applications requiring accurate position and/or force control. In formulating this model, the authors propose a generalized Maxwell resistive capacitor as a lumped-parameter causal representation of rate-independent hysteresis. Model formulation is validated by comparing results of numerical simulations to experimental data. Validation is followed by a discussion of model implications for purposes of actuator control.

\section{Actuation for Interactive Micromanipulation}

Micromanipulator applications, and in particular dynamically interactive micromanipulation, require control actuators that can provide both accurate position tracking performance in addition to robustly stable force control $[2,7,13]$. These objectives are significantly compromised by the presence of backlash and Coulomb friction in the control plant $[4,14,16]$, the effects of which are exaggerated at small scales [5]. Since piezoelectric stack actuators are monolithic and have no sliding or rolling parts, they exhibit no significant mechanical stiction or backlash. Additionally, a typical lead-zirconate-titanate (PZT) piezoelectric stack actuator can perform step movements with a resolution on the order of a nanometer, has a bandwidth on the order of a kilohertz, and can provide mechanical power on the order of several watts. Consequently, piezoelectric ceramics are well suited for use as precision microactuators for micropositioning devices, and due to the absence of Coulomb friction and backlash, are especially well suited for use in dynamically interactive micromanipulation applications.

The use of PZT stack actuators for accurate and stable control of manipulator position and/or force is greatly facilitated by model-based control system analysis and design. The purpose of the model presented herein is to map the relationship between voltage and charge at the electrical port of the PZT to force and displacement at the mechanical port in a lumped parameter form that can be represented by a set of ordinary differential equations.

Goldfarb is an assistant professor in the Department of Mechanical Engineering at Vanderbilt Universin: and can be reached at Box 1592 Station B. Nashville TN. 37235, or via email a goldfarb@vu.se.vanderbilt.edu. Celanuvic was a student in the Deparrment of Mechanical Engineerins at Vanderbilt at the time the nork was performed, and is prisemly wish the Department of Electrical Engineering at Virginia Polytectmic University. A version of this article appears in the prreceeding.s of the 1996 IEEE International Conference an Robotics and Ausomation. Minmeapelis, MN, April 1996.
This type of formulation provides both general insight into PZT behavior as well as a specific causal mathematical representation for purposes of model-based control system analysis and design.

The Anatomy of a Piezoelectric Stack Actuator

Piezoelectric ceramics transduce energy between the electrical and mechanical domains. Application of an electric field across the ceramic creates a mechanical strain, and in a similar manner, application of a mechanical stress on the ceramic induces an electrical charge. The fundamental component of a piezoelectric stack actuator is a wafer of piezoelectric material sandwiched between two electrodes. Prior to fabrication, the wafer is polarized uniaxially along its thickness, and thus exhibits significant piezoelectric effect in this direction only. A typical piezoelectric stack actuator is formed by assembling several of the wafer elements in series mechanically and connecting the electrodes so that the wafers are in parallel electrically, as illustrated in Fig. 1. The nominal quasi-static behavior of a piezoelectric stack actuator is a steady-state output displacement that is monotonically related to the voltage input.

Existing Descriptions of Piezoelectric Behavior :

The most widely recognized description of piezoelectric ceramic behavior was published by a standards committee of the IEEE Ultrasonics, Ferroelectrics, and Frequency Control Society, originally in 1966 and most recently revised in 1987 [15]. This committee formulated linearized constitutive relations describing piezoelectric continua which form the basis for the model of piezoelectric behavior that is presently in general use. The linearized constitutive relations are typically represented in a compressed matrix notation as follows:

$$
\begin{aligned}
& S_{p}=s_{p q}^{E} T_{q}+d_{k p} E_{k} \\
& D_{i}=d_{i \psi} T_{\psi}+E_{i k}^{T} E_{k}
\end{aligned}
$$

where $S$ represents the strain tensor, $s^{E}$ is the elastic compliance matrix when subjected to a constant electrical field, $T$ represents the stress tensor, $d$ is a matrix of piezoelectric material constants, $E$ is the electric field vector. $D$ the electric displacement vector, and $\varepsilon^{T}$ the permittivity measured at a constant stress. The compressed notation eliminates redundant terms by representing the symmetric stress and strain tensors with single column vectors that incorporate elements representing both the diagonal and ofi-diagonal tensor cerms. These equations essentially state that the matterial strain and clectrical displacement (charge per unit area) exhibited by a nicacelectric leramic are both linearly affecled by the mechanical stress and electrical ficld to which the 


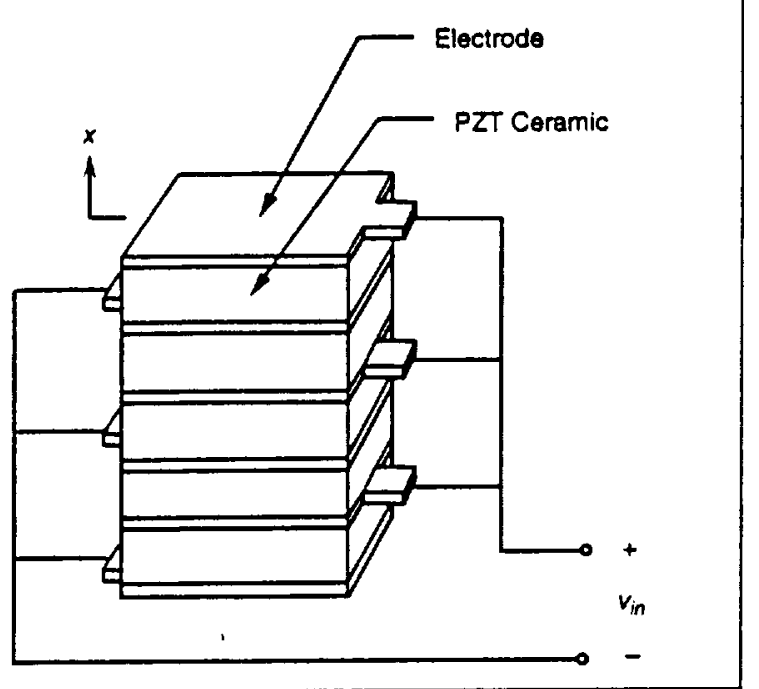

Fig. 1. Illustration of a piezoelectric stack actuator.

ceramic is subjected. Aside from the awkward notation, these linearized constitutive relations fail to explicitly describe the nonlinearities that are present in all piezoelectric ceramics. Additionally, the derivation assumes a purely conservative energy field, and thus fails to describe the dissipative behavioral aspects of the ceramic.

Several authors have utilized these linearized constitutive relations to derive a piezoelectric actuator model $[6,10,12]$. Given the aforementioned limitations of the IEEE model, however, the resulting descriptions are too approximate for use in developing accurate position or force control. Leigh and Zimmerman took an iterative algorithmic approach to describing the nonlinear behavior of piezoelectric ceramics [11]. Though capable of describing the hysteretic behavior of piezoelectrics, this approach is neither real-time nor energy-based. and thus provides little physical insight into actuator behavior and has limited use with respect to model-based control system design. Jouaneh and Tian incorporated a time-dependent nonlinear state variable, a method originally developed by Bouc for a structures application, to describe the nonlinear voltage/displacement behavior of piezoelectric actuators [1,8]. The model of Jouaneh and Tian is nicely posed, but as an electromechanical model is incomplete, since it represents both the electrical and mechanical ports of the PZT as signal ports (i.e., the model implicitly assumes no dynamic interaction with the environment). Utilizing piezoelectric ceramic as an actuator explicitly requires that it perform work on its environment. An accurate behavioral description must therefore characterize the input and output ports of the PZT as power ports, and must therefore describe relationships involving mechanical force and electrical charge as well as electrical voltage and mechanical displacement.

The model presented herein offers a complete behavioral description that is based neither on the IEEE constitutive relations, nor on algorithm, but rather is formulated by describing the observed static and dynamic behavior of a piezoelectric actuator with a lumped-parameter energy-based representation.

\section{Model Formulation}

Since piezoceramic is a known dielectric, one would expect a PZT stack actuator to exhibit capacitive behavior. The electrical behavior of the actuator, however, is significantly more complex. For purposes of controller design, one of the most inconvenient aspects of the actuator behavior is the rate-independent hysteresis exhibited between voltage and displacement as well as between force and displacement, as shown in Fig. 2. If not specifically addressed, this type of behavior can cause closedloop limit cycling and possibly instability. Experimental observation indicates that the rate-independent hysteresis exhibited in Fig. 2 is not present between the endpoint displacement of the

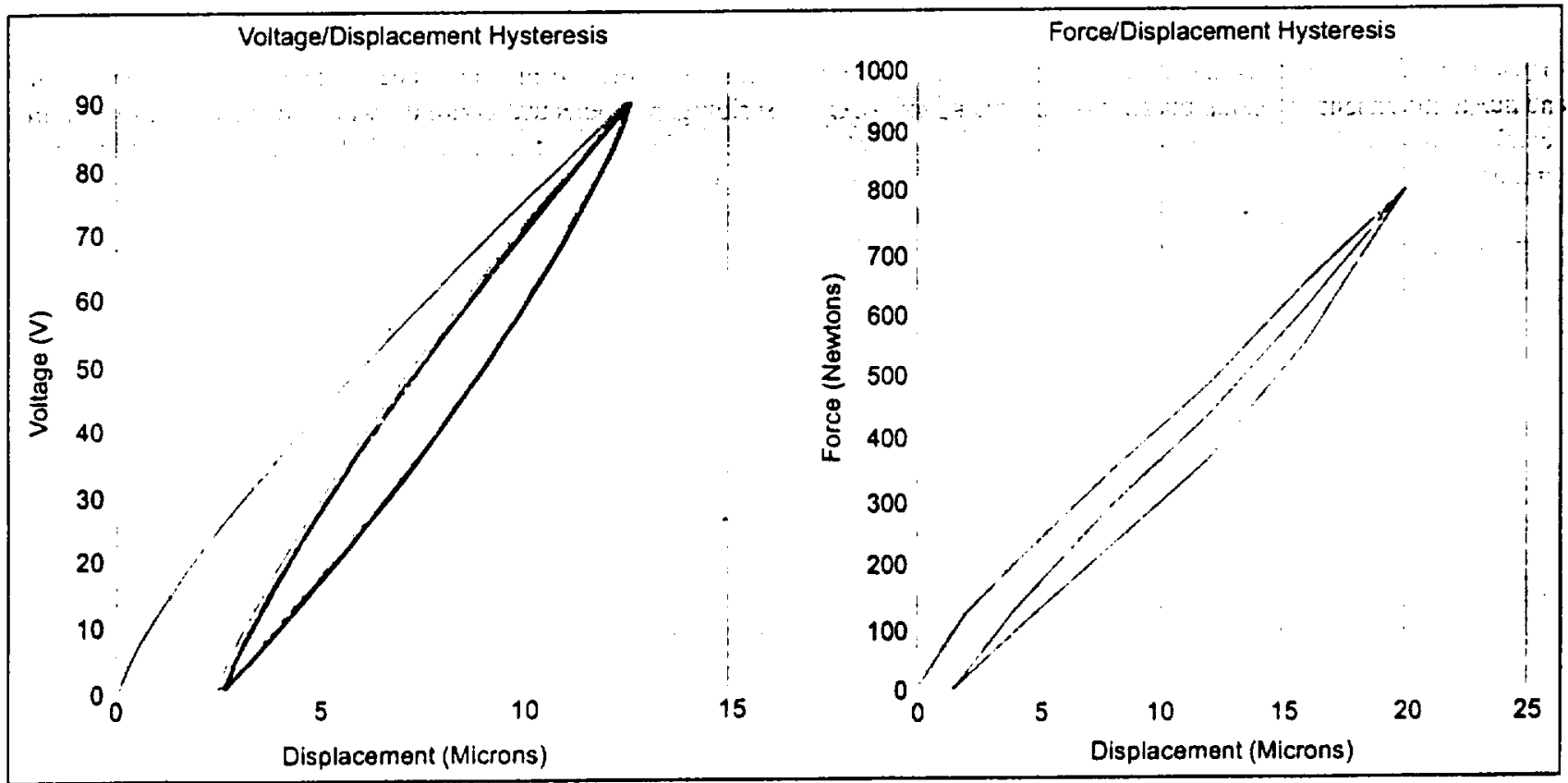

Fig. 2. Measured quasi-static relationships between applied voltage and endpoint displacement and between applied force anul endpoint displacement. 


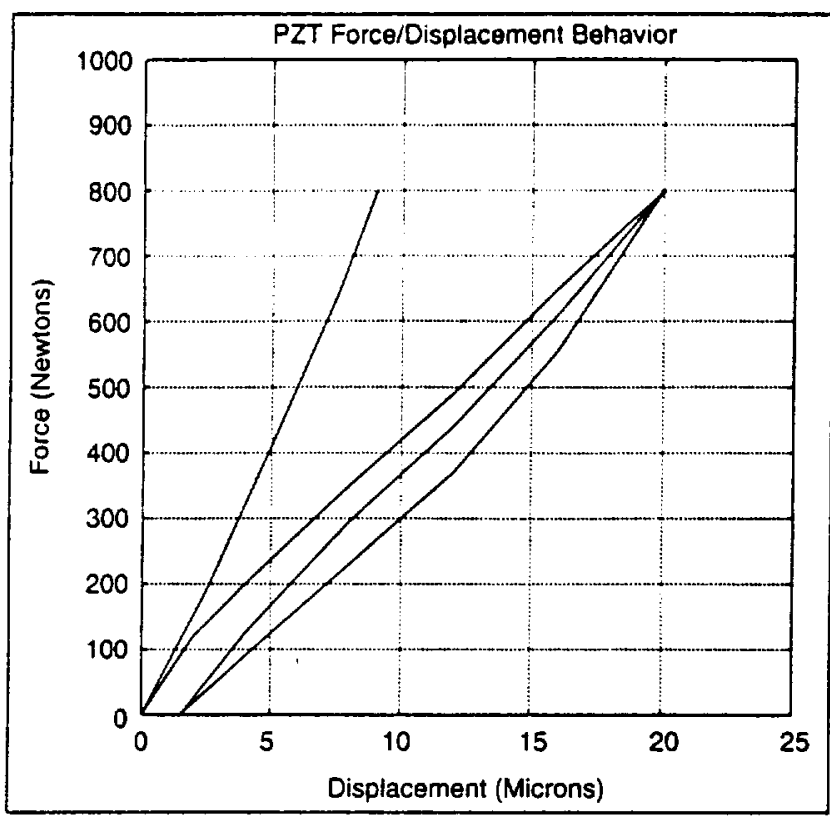

Fig. 4. Quasi-static force displacement relationship for a PZT stack actuator with open leads (dashed line) and with shorted leads (solid line).

\section{Modeling Hysteresis}

Generalized hysteretic behavior is characterized by two variables related by a non-single-valued function, as illustrated by the voltage/displacement relationship in Fig. 2. Examples of such behavior include the relationship between the stress and strain in the elastic-plastic deformation of a material, and the relationship between magnetic field strength and flux density in a hard magnetic material. In these and in most physical systems, this hysteretic behavior is the result of energy storage that is fundamentally coupled to rate-independent dissipation. Mechanically, such behavior could be modeled by the combination of an ideal spring, which represents pure energy-storage, coupled to a pure Coulomb friction element, which represents rateindependent dissipation. This analogy is the basis for describing the static hysteresis exhibited by the piezoelectric actuator. Fig. 5 illustrates a single elasto-slide element which consists of a massless linear spring and a massless block that is subjected to Coulomb friction. The constitutive behavior of this element can be described by:

$$
F=\left\{\begin{array}{c}
i\left(x-x_{h}\right) \text { if } \mid k\left(x-x_{b} \mid<f\right. \\
f \operatorname{sgn}(\dot{x}) \text { and } x_{b}=x-\frac{f}{k} \operatorname{sgn}(\dot{x}) \text { else }
\end{array}\right.
$$

where $x$ is the input displacement, $F$ is the output force, $k$ is the stiffiness of the spring. $f=\mu N$ is the breakaway friction force of the block. and $x_{b}$ is the position of the block. For a displacement input of sufficient amplitude, the relationship between the applied foree and the endpoint displacement will exhibit rudimentary hysteretic behavior. If several of these elast(o-slide elements are put in parallel, each subjected to an incrementally larger normal force (i.e., each has an incrementally larger breakaway force), the simple relationship of Fig. 5 becomes a piecewise lin-

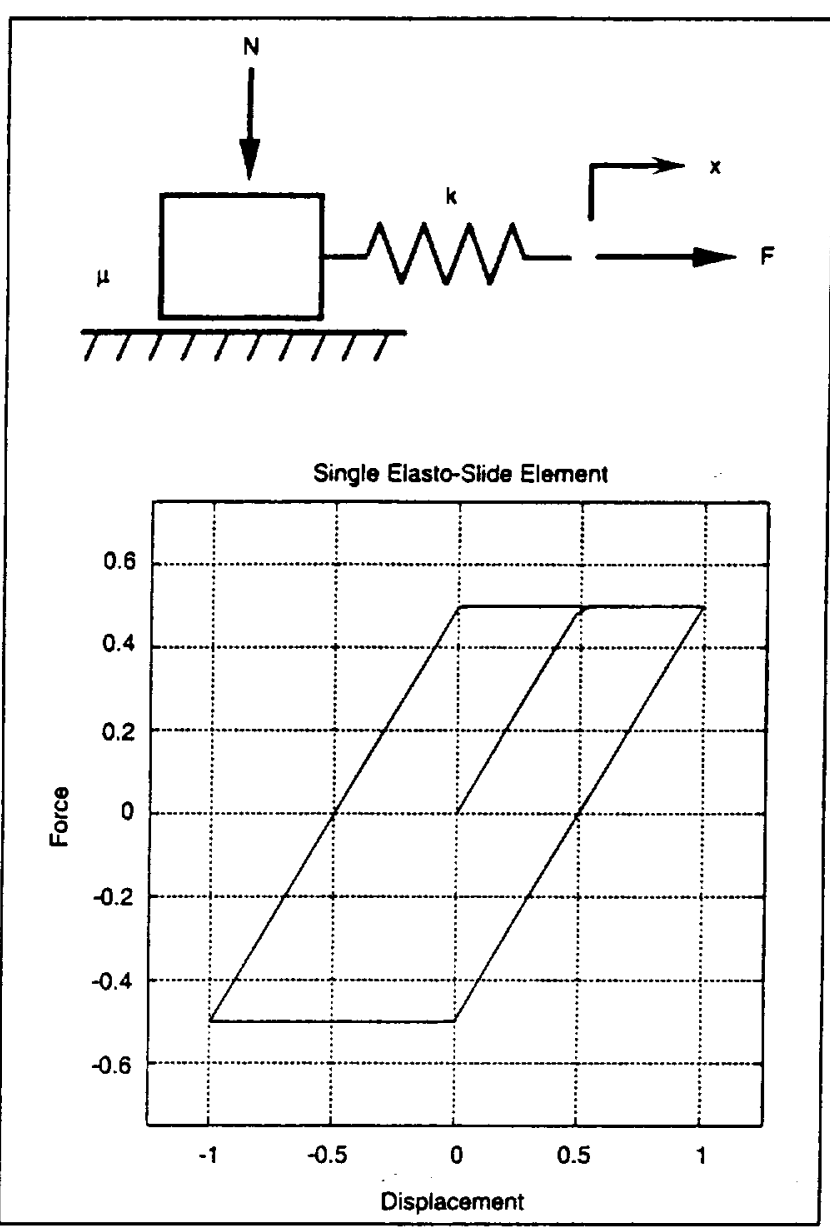

Fig. 5. Force-displacement behavior of a single elasto-slide element consisting of a massless linear spring and a massless block subjected to Coulomb friction.

ear approximation of rate-independent hysteresis, as illustrated in Fig. 6. This construction was initially formulated by the mathematician and physicist James $C$. Maxwell in the mid$1800 \mathrm{~s}$, and in the limit as the number of elasto-slide elements becomes infinite, the model is referred to as Generalized Maxwell Slip [9]. The constitutive behavior of the system in Fig. 6 containing $n$ elasto-slide elements can be described by:

$$
F_{i}=\left\{\begin{array}{c}
k_{i}\left(x-x_{b_{i}}\right) \text { if }\left|k_{i}\left(x-x_{b_{i}}\right)\right|<f_{i} \\
f_{i} \operatorname{sgn}(\dot{x}) \text { and } x_{b_{i}}=x-\frac{f_{i}}{k_{i}} \operatorname{sgn}(\dot{x}) \text { else }
\end{array}\right.
$$

$$
F=\sum_{i=1}^{n} F_{i}
$$

where $x$ is the input displacement, $F$ is the output force, and $F_{i}, k_{l}$, $f_{i}$, and $x_{\mathrm{A}}$ are the output force, spring stiffness, breakaway force, and block position. respectively, of the $i^{\text {th }}$ elasto-slide element.

The Maxwell model of rate-independent hysteresis can be parameterized directly from a piecewise linear fit of the rising curve of the hysteresis from a relaxed state, as illustrated in Fig. 7. Note that complete parameterization of the entire hysteretic 


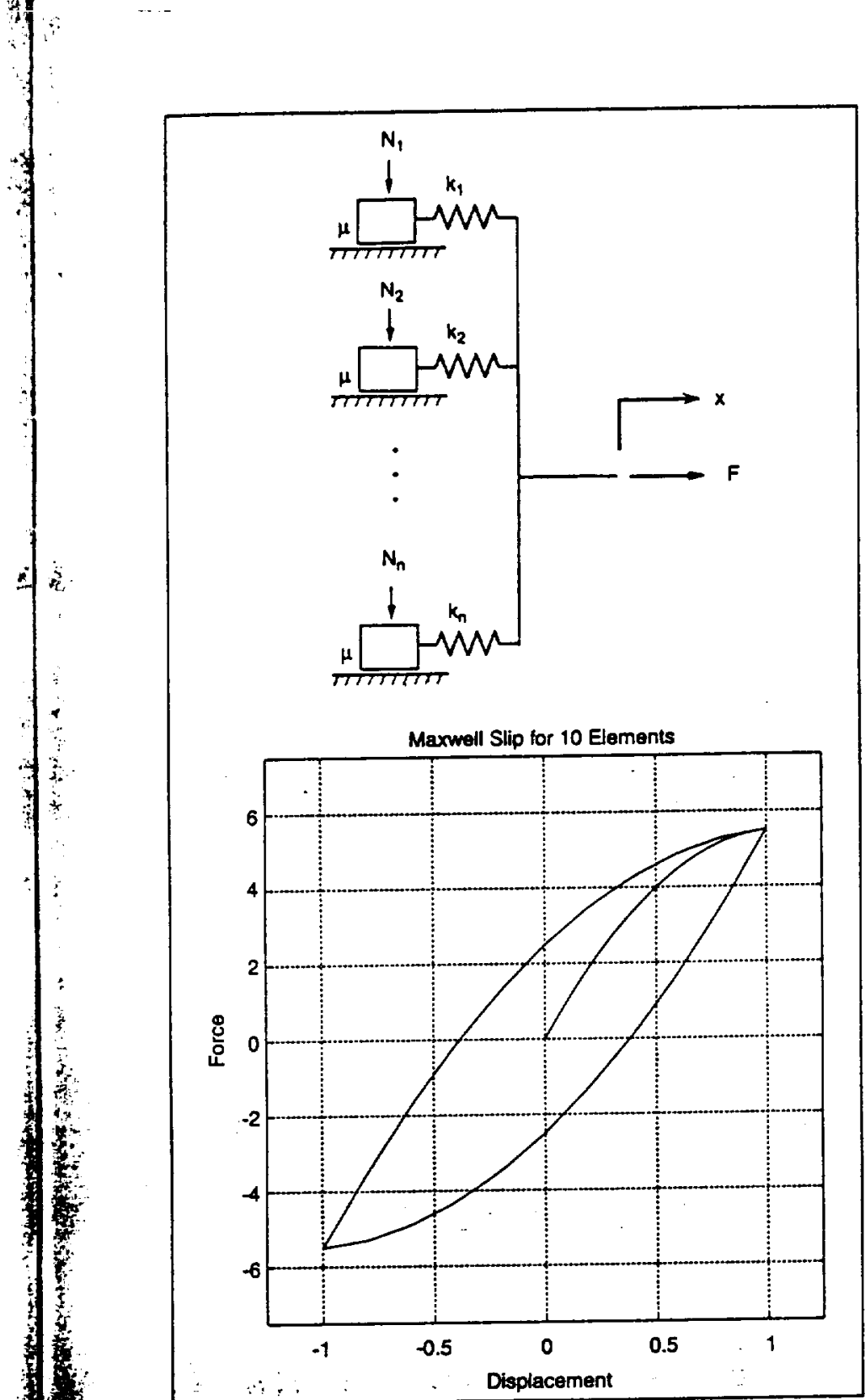

Fig. 6. Force-displacement behavior of several elasto-slide elements in parallel, each subjected to increasing normal forces.

behavior requires fitting only the initial rising curve. A rising curve fit by $n$ linear segments will require $n$ elasto-slide elements, and will thus require $2 n$ parameters. For a curve fit by $n$ linear segments, the slope of the $\mathrm{j}^{\text {th }}$ segment (the localized stiffness) is given by:

$$
s_{j}=\sum_{i=1}^{n} k_{i}
$$

where $k_{i}$ represents the stiffness of the $i^{\text {th }}$ spring in the Maxwell model. This can be expressed in a matrix form as:

$$
\mathbf{s}=\mathbf{A} \mathbf{k}
$$

where s is a $n \times 1$ vector of the segment slopes, $A$ is an $n \times n$ upper triangulur matrix of ones, and $k$ is an $n \times \mid$ vector of Maxwell

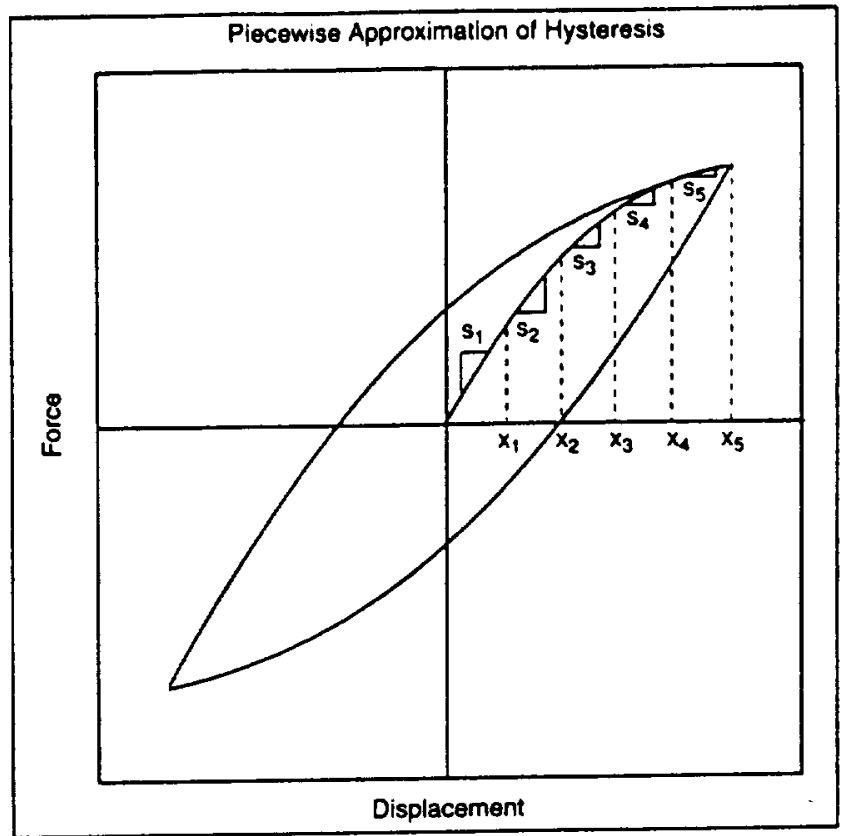

Fig. 7. Parameterizing the Marwell model of rate-independent hysteresis. Complete parameterization requires piecewise linear fit of the rising curve of the hysteresis, taken from an initially relaxed state.

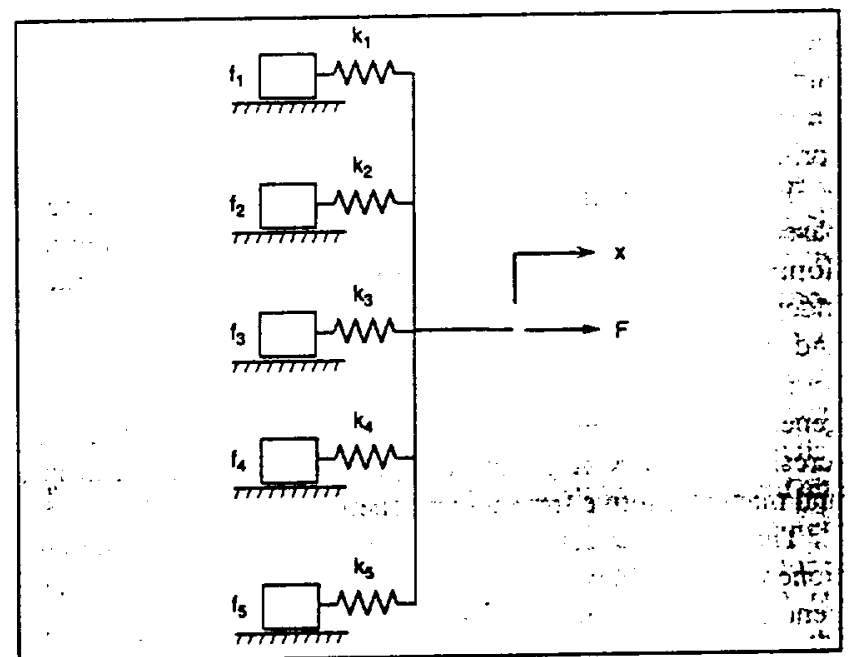

Fig. 8. Maxwell model parameters corresponding to hysteresis and piecewise linear fit of Fig. 7.

model spring stiffness, as illustrated in Fig. 8. Rearranging gives an explicit expression for the spring stiffnesses:

$$
\mathbf{k}=\mathbf{A}^{\mathbf{1}} \mathbf{s} \text {. }
$$

The location of each respective segment, defined by $x_{j}$ in the five segment fit of Fig. 7, can be expressed as:

$$
x_{1}=\frac{f_{1}}{k_{j}}
$$




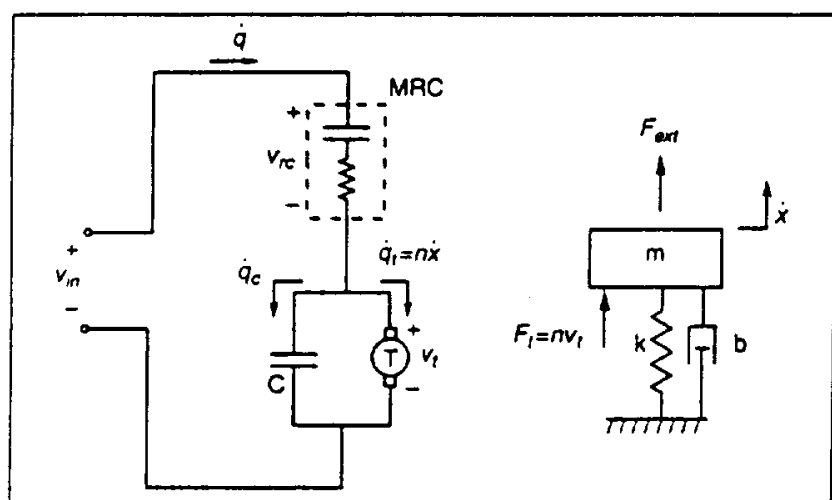

Fig. 9. Schematic representation of the PZT stack actuator model. The capacitor and resistor contained within the MRC element are both nonlinear elements.

where $f_{j}$ is the breakaway friction force of the $j^{\text {th }}$ block in the Maxwell model. Rearranging gives an explicit expression for the breakaway forces:

$$
\mathbf{f}=\mathbf{K} \mathbf{x}
$$

where $\mathrm{f}$ is an $n \times \mathrm{l}$ vector of the breakaway forces, $\mathrm{K}$ is an $n \times n$ diagonal matrix of the spring stiffnesses, and $x$ is an $n \times 1$ vector of the segment locations as shown in Fig. 7. Note that since the Maxwell model is a piecewise linear approximation, increasing the number of blocks will increase the model accuracy. The number of elasto-slide elements, however, has no bearing on the order of the model, since the blocks are all massless and the springs are in parallel.

Though ostensibly a mechanical formulation, the energybased constitutive relations of the Maxwell slip model are not domain-specific, and can therefore represent any rateindependent hysteretic relationship between a generalized force and generalized displacement in a lumped parameter causal form. Consequently, in addition to force and displacement, the generalized Maxwell model can represent rate-independent hysteresis between voltage and charge, temperature and entropy, and magnetomotive force and magnetic flux.

The PZT stack actuator model resulting from the aforementioned observations is shown in schematic form in Fig. 9. The generalized Maxwell resistive capacitance, which is represented by the MRC element, resides in the electrical domain and therefore relates the element's electrical voltage to charge. The PZT model has two ports of interaction, a voltage-current port on the electrical side and a force-velocity port on the mechanical side. With respect to the mechanical side of the transformer, since the actuator model is concerned only with endpoint displacement in a frequency band within the first mechanical mode of vibration, the piezoelectric stack is assumed to have a lumped mass and a linear material stiffness and damping. The behavior of the actuator is therefore described by:

$$
\begin{aligned}
& q=T x+C v_{1} \\
& v_{1}=v_{m}+v_{n}
\end{aligned}
$$

$$
\begin{gathered}
v_{\tau}=\operatorname{mrc}(q) \\
F_{1}=T v_{1} \\
m \ddot{x}+b \dot{x}+b x=F_{1}+f_{r v}
\end{gathered}
$$

where $q$ is the total charge in the ceramic, $T$ is the electromechanical transformer ratio, $x$ is the stack endpoint displacement, $C$ is the linear capacitance in parallel with the transformer, $v_{t}$ is the back-emf from the mechanical side, $v_{i n}$ is the actuator input voltage, $v_{r}$ is the voltage across the Maxwell capacitor (which as indicated by Equation ( 13 ) is a function of $q$ ), $F_{t}$ is the transduced force from the electrical domain, $m, b$, and $k$ are the mass, damping, and stiffness of the ceramic, and $F_{e x t}$ is the force imposed from the external mechanical load. The function of equation (13) relates the voltage across the MRC element to the charge in the ceramic as follows:

\begin{tabular}{|l|l|l|}
\hline \multicolumn{2}{|c|}{$\begin{array}{c}\text { Table 1. Model Parameters Utilized in Simulation of the NEC } \\
\text { Model \#AE0505D16 Piezoelectric Actuator }\end{array}$} \\
\hline Model Parameter & Symbol & Numerical Value \\
\hline Mass & $m$ & $0.00375 \mathrm{~kg}$ \\
\hline Stiffness & $k$ & $6 \times 10^{6} \mathrm{~N} / \mathrm{m}$ \\
\hline Damping & $b$ & $150 \mathrm{~N}-\mathrm{s} / \mathrm{m}$ \\
\hline Linear Capacitance & $C$ & $1.2 \times 10^{-6} \mathrm{~F}$ \\
\hline Transformer Ratio & $T$ & $10 \mathrm{C} / \mathrm{m}$ \\
\hline
\end{tabular}

$$
V=\left\{\begin{array}{c}
\frac{\left(q-q_{q_{1}}\right)}{C_{i}} \text { if }\left|\frac{\left(q-q_{q}\right)}{C_{i}}\right| \\
v_{i} \operatorname{sgn}(i) \text { and } q_{b_{i}}=q-C_{i} v_{i} \operatorname{sgn}(i) \text { else }
\end{array}\right.
$$

where $q$ is the charge in the ceramic, $v_{r}$ is the voltage across the Maxwell capacitor, and all other variables are the electrical analogs of those given in Equations (4) and (5).

\section{Simulation and Experiment}

The model was parameterized for a commercially available piezoelectric stack actuator (NEC model \#AE0505D16). This actuator operates at input voltages between zero and 150 volts (in the direction of wafer polarization), which corresponds to an endpoint displacement range of approximately 20 microns. Upon measuring the mass $m$ of the PZT, the mechanical stiffness $k$ and damping $b$ can be determined by observation of the charge-displacement dynamics that are shown in Fig. 3. Meas: urement of the open-lead stiffness and knowledge of the DC gain between charge and displacement are sufficient to determine the linear electrical capacitance $C$ and the transformer ratio $T$. The 


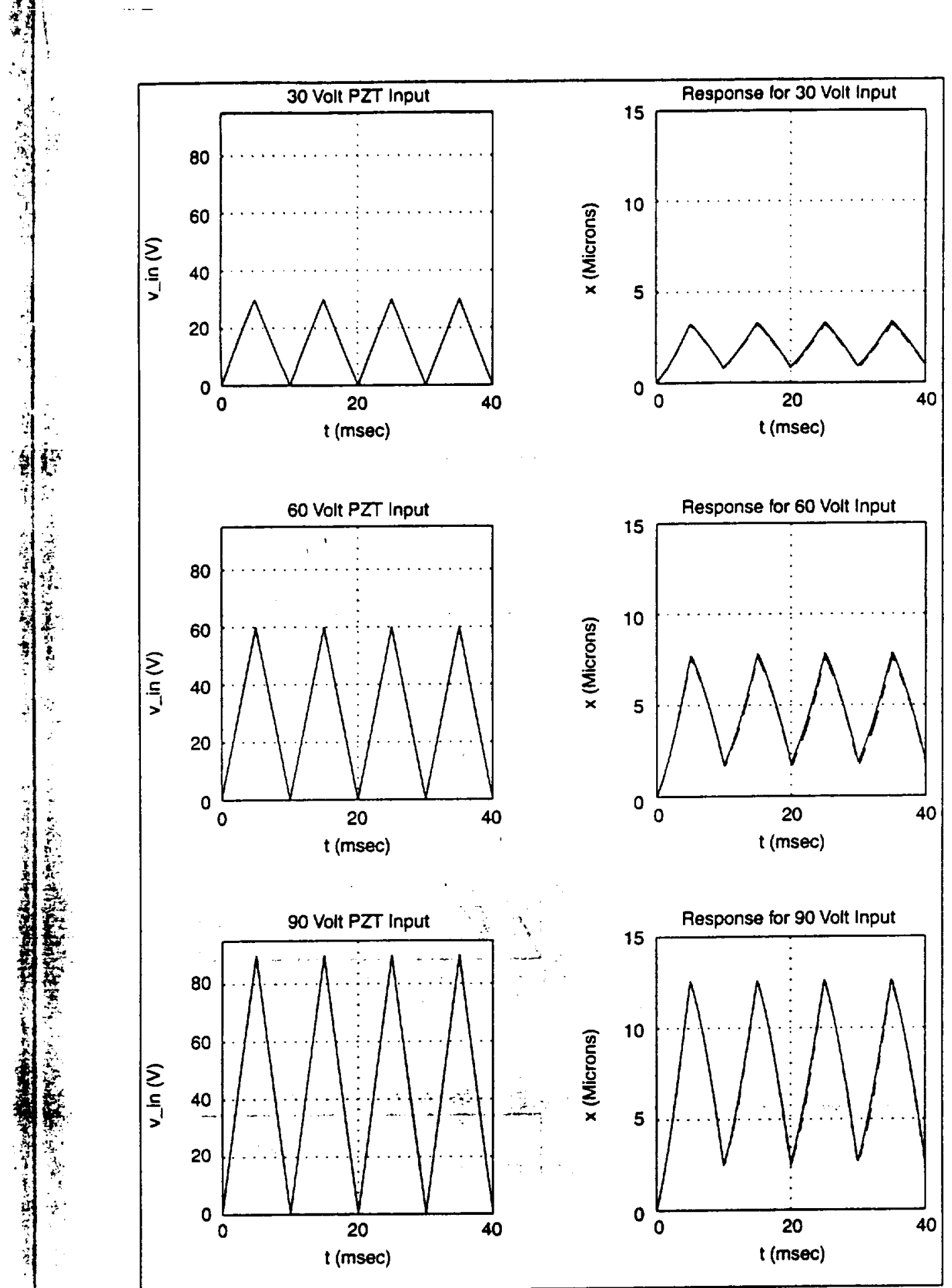

Fig. 10. Measured (solid line) and simulated (dashed line) endpoint displacement response of the $P Z T$ to a 90 volt $100 \mathrm{~Hz}$ triangle-wave input. The difference between the measured and simulated data is difficult to discern.

model parameters utilized for the simulations that follow are given in Table 1.

The Maxwell capacitor parameters, as given in Table 2, were determined by piecewise linear fit of the measured voltage versus charge data. More specifically, the electrical parameters of Equations (16) and (17), $C_{l}$ and $v_{1}$, were found using the methods of Equations (6) through (10), where each stiffness of
Equition (8) is replaced with the inverse of a capacitance and each breakawaly force of Equation (10) is replaced with an analogous saturation voltage:

$$
\begin{aligned}
& \frac{1}{C_{1}}=k \\
& \nu_{1}=f_{1} .
\end{aligned}
$$

The parameters defining the Maxwell capacitor for the simulations presented herein are given in Table 2. As indicated by the table, these simulations were run with ten elasto-slide elements in the generalized Maxwell resistive capacitor. As previously stated, the Maxwell model is essentially a piecewise linear approximation of rateindependent hysteresis where the number of elasto-slide elements corresponds to the number of linear segments. Increasing the number of blocks therefore increases the model accuracy. The number of blocks required to accurately model a given hysteresis thus depends on the curvature of the hysteresis and the required degree of model accuracy. A real-time application may be better served by fewer elements; since the number of elements does not affect the order of the model, however, the increased computational overhead from added elements is minimal. .:

Fig. 10 shows the measured and simulated endpoint displacement response of the modeled actuator to a 30,60 , and 90 volt $100 \mathrm{~Hz}$ triangle-wave voltage input in the absence of an external mechanical load. As indicated by the plots, the model faithfully represents the voltage input to endpoint displacement output behavior of the PZT actuator. Note in the plots that the displacement output evinces distortion on both the rising and falling slopes and maintains an amplitude-dependent offset. Further observation indicates that this is not typical dynamic distortion. There is no discernible phase lag between the input and output, as indicated by the relative positions of the waveform peaks, and there are no signilicant liflering effects. evidenced by the fact that the peaks are incither rounded nor oflerwise distorted. These observations indicate the existence of a rate- 


\begin{tabular}{|l|l|l|}
\hline \multicolumn{3}{|c|}{$\begin{array}{c}\text { Table 2. Generalized Maxwell Capacitor } \\
\text { Parameters Utilized in the Model* }\end{array}$} \\
\hline Element & Stiffness & Break Force \\
\hline$i$ & $k_{i}\left(/ / C_{i}\right)$ & $f_{i}\left(v_{i}\right)$ \\
\hline 1 & 2.0 & 0.2 \\
\hline 2 & 0.6 & 0.3 \\
\hline 3 & 0.3 & 0.3 \\
\hline 4 & 0.26 & 2.6 \\
\hline 5 & 0.06 & 0.9 \\
\hline 6 & 0.1 & 2.0 \\
\hline 7 & 0.05 & 1.5 \\
\hline 8 & 0.03 & 1.2 \\
\hline 9 & 0.1 & 7.0 \\
\hline 10 & 0.5 & 80.0 \\
\hline$*$ The stiffnesses and breakaway forces correspond with the me- \\
chanical schematic of Fig. 6.
\end{tabular}

independent hysteresis. This hysteretic behavior is clearly displayed in Fig. 11, which shows both the measured and simulated data of Fig. 10 plotted as voltage input versus displacement output. Any dynamic system will exhibit an ellipsoid-shaped inputoutput hysteresis, provided the input is of sufficient frequency to create discemible phase lag in the output. This type of dynamic hysteresis, characterized by a smooth curve relating the output to input, is a result of phase lag between the input and output (such as that shown in Fig. 3). The rate-independent hysteresis of Fig. 10 , however, is distinguished from a dynamic hysteresis by the distinct discontinuities exhibited at both extremes. Model accuracy is additionally demonstrated by the data of Fig. 12, which shows the measured and simulated response of the actuator to a linearly decaying 100 -hertz sinusoidal voltage input.

Fig. 13 shows the simulated displacement response to a $10 \mathrm{~Hz}$ sinusoidal external force for cases with the electrical leads open and shorted. The simulated behavior accurately reflects the quasistatic measured behavior illustrated by Fig. 4 . In addition to providing a structure for numerical simulation, the lumped-parameter model also provides insight into how the electrical properties of the PZT reflect into the mechanical domain. As shown by the measured and simulated data of Figs. 4 and 13, respectively, the PZT actuator exhibits significantly greater stiffness when the leads are open than when the leads are shorted. This change in stiffness can be demonstrated by observing that both the linear and the Maxwell capacitors in the electrical domain reflect as stiffnesses in the mechanical domain. This is similar to the electrical resistance of a DC motor appearing as mechanical damping when the motor leads are shorted. Linearizing the Maxwell capacitor and deriving expressions for mechanical stiffness in both the open and shored-lead contigurations yields

$$
k_{10}=k+\frac{T^{2}}{C}
$$

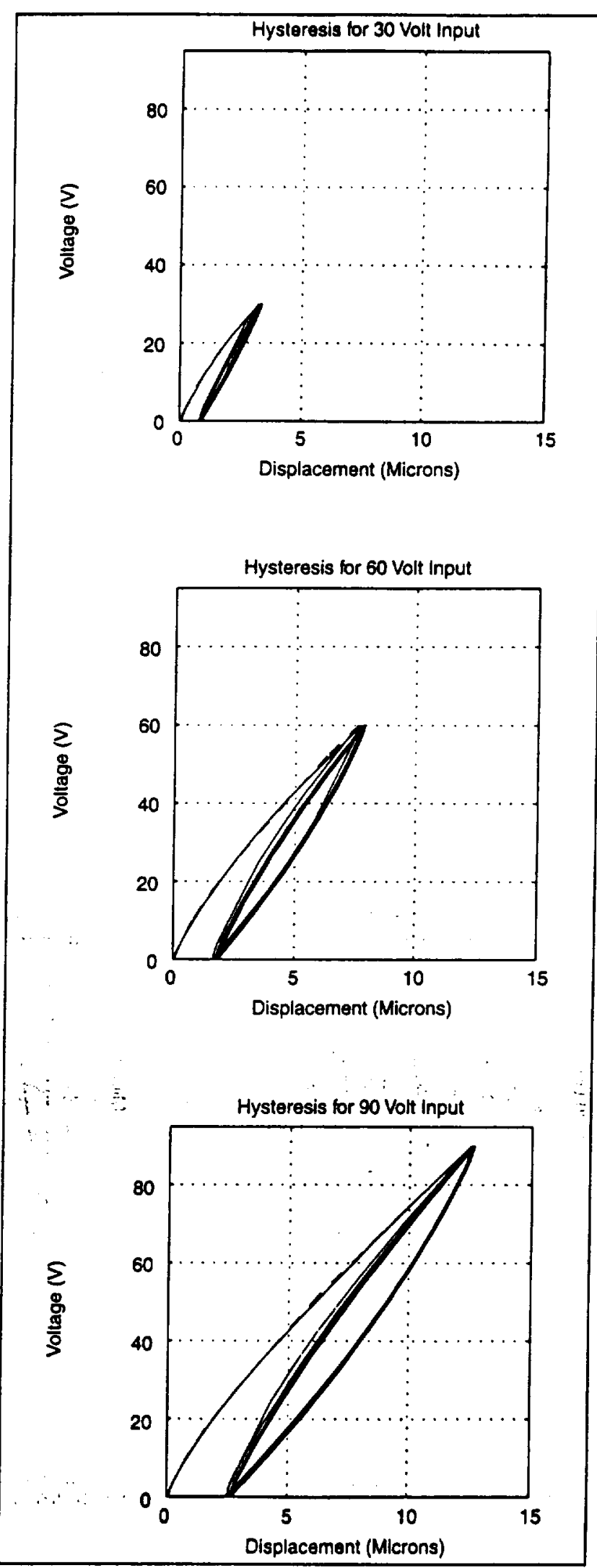

Fig. II. Measured (solid line) and simulated (dashed line) voliage versus displacement for a 90 volt $100 \mathrm{~Hz}$ triangle-wave voliage input. The difference benween the measured and simulated dasa is difficult to discern. 


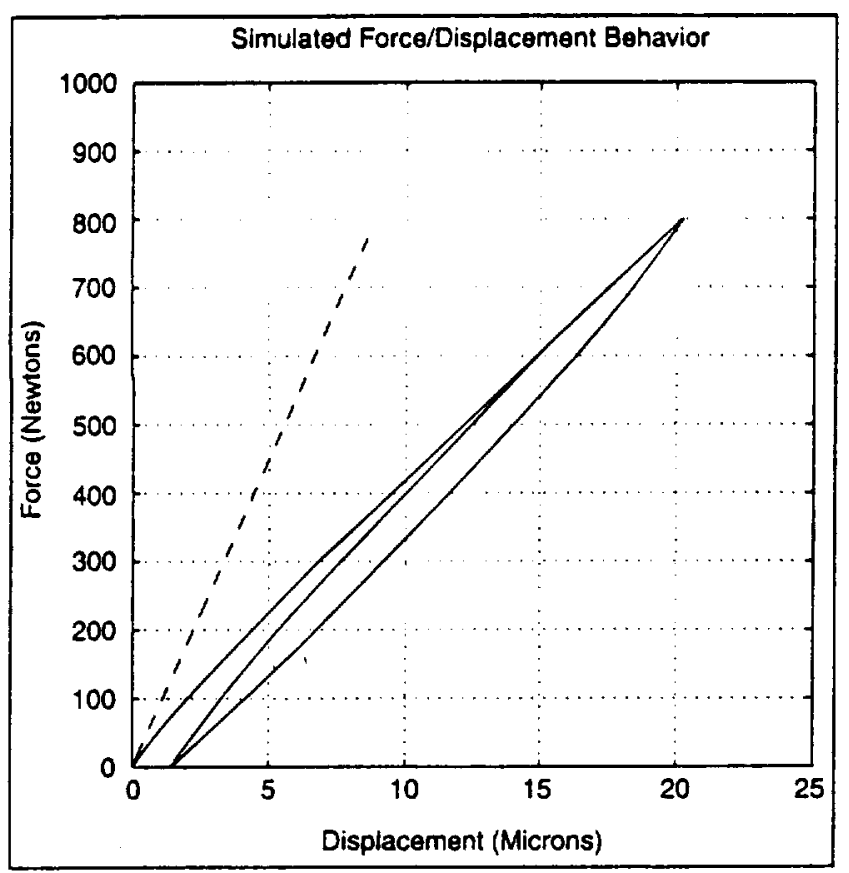

Fig. 13. Simulated PZT behavior showing force versus endpoint displacement for a $10 \mathrm{~Hz}$ sinusoidal extemal force input for cases with the electrical leads open (dashed line) and shorted (solid line).

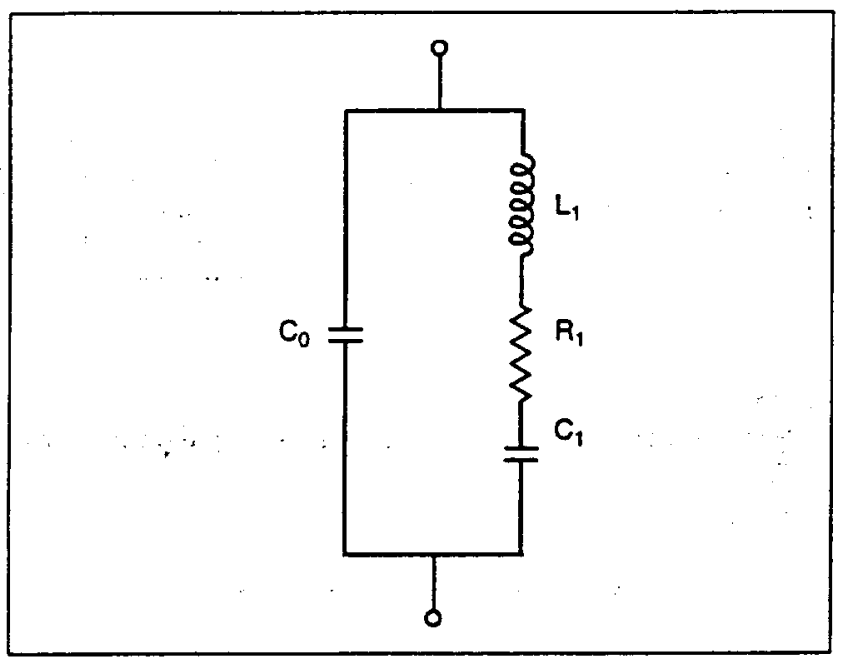

Fig. 14.IEEE lumped parameter cincuit model of PZT near isolated resonance.

$$
k_{s}=k+\frac{r^{2}}{C_{m}+C}
$$

where $k_{1}$ is the open lead stiffness, $k_{x}$ is the shorted lead stiffness, $k$ is the mechanical domain stiffness, $C$ is the lincar electrical capacilance. $T$ is the transformer ratio, and $C_{m}$ is the linearized Maxwell cupacitor. The inclusion of $C_{m}$ in the second term of the latter expression clearly indicates the decreased shorted lead stilliness.
Comments on the IEEE Resonance Model

In addition to formulating the previously described linearized constitutive relations for piezoelectric continua, the IEEE standards publication [I5] also offers a circuit representation of a PZT near an isolated resonance, shown in Fig. 14. The standards publication does not relate the resonance circuit to the constitutive relations and does not offer any explanation for the presence of inductive or resistive effects in what is, according to the constitutive equations, a multi-port capacitive medium. The model derived herein elucidates the structure of the resonance model and accounts for the inductive and resistive elements. The inductor, resistor, and capacitor $\left(L_{1}, R_{1}\right.$, and $C_{1}$, respectively) in the IEEE resonance circuit of Fig. 14 are not fundamentally electrical behaviors, but rather are the apparent behavior of the first mode of mechanical vibration, as seen through the electrical port. Reflecting the mechanical elements of the lumped parameter model to the electrical side gives circuit element values of

$$
\begin{aligned}
& L_{1}=\frac{m}{T^{2}} \\
& R_{1}=\frac{b}{T^{2}} \\
& C_{1}=\frac{T^{2}}{k} \\
& C_{0}=C .
\end{aligned}
$$

This would indicate that driving a typical mass-springdamper external load would effectively increase $L_{1}$ and $R_{1}$, decrease $C_{1}$, and leave $C_{0}$ unaffected.

\section{Implications for Actuator Control}

Several analogies can be drawn between control of a DC permanent magnet motor and control of a PZT stack actuator. First, controlling charge into a PZT actuator is in many ways analogous to controlling current into a DC motor. The relationship between input current and output displacement of a DC motor can be characterized by linear second-order dynamics (one pole serving as a pure integrator). In a similar manner, the relationship between input charge and output displacement of a PZT stack can also be characterized by linear second-order dynamics. Second, just as the output shaft torque is directly proportional to input current in a DC motor when the shaft is locked, the output force of a PZT stack is similarly proportional to input charge when the stack is locked. Continuing this analogy, controlling a DC motor with voltage as input introduces the first-order dynamics of the stator coil inductance and resistance into the system. In a similar manner, controlling a PZT stack with voltage as input introduces the first-order dynamics of the Maxwell resistive capacitor into the system, which unlike the inductor/resistor dynamics of the DC motor, are nonlinear.

\section{Summary and Conclusions}

The model presented accuritcly represents the behavior of a piezoclectric stack actuator in a lumped-parameter real-time rep- 
resentation, and can therefore be utilized for purposes of modelbased control analysis and design. The static hysteresis evinced by the PZT actuator was identified as energy storage coupled io ratc-independent dissipation, and was faithfully represented by a generalized elasto-slip model. Despite the presence of this nonlinearity, the relationship between charge delivered to the PZT and endpoint displacement of the stack was observed to hasc simple second-order linear characteristics. Design and analysis of closed-loop actuator control can therefore be achieved either by incorporating nonlinear techniques with actuator voltage as command, or by utilizing linear techniques with actuator charge as command.

\section{Acknowledgment}

This work was supported by NASA Grant No. NAGW-4723 and by the Vanderbilt University Research Council. The authors gratefully acknowledge this support.

\section{References}

[1] R. Bouc, "Forced Vibration of Mechanical Systems with Hysteresis," Proceedings of the Fourth Conference on Nonlinear Oscillation. Prague. Czechoslovakia, 1967.

[2] J.E. Colgate and N. Hogan, "Robust Control of Dynamically Interacting Systems," International Journal of Control, 48(1):65-88. 1988.

[3] R. Comstock. "Charge Control of Piezoelectric Actuators to Reduce Hysteresis Effects." United States Patent \#4.263,527. Assignee: The Charles Stark Draper Laboratory, Cambridge, MA, 1981.

[4] A. Gogoussis and M. Donath, "Determining the Effects of Coulomb Friction on the Dynamics of Bearings and Transmissions in Robot Mechanisms." ASME Joumal of Mechanical Design, vol. 115, no. 2, pp. 231-240, 1993.

[5] M. Goldfarb and N. Celanovic, "Minimum Surface Effect Microgripper Design for Force-Reflective Telemanipulation of a Microscopic Environment," Proceedings of the ASME International Mechanical Engineering Conference and Exposition, November 1996.

[6] N.W. Hagood, W.H. Chung, and A. von Flotow, "Modelling of Piezoelectric Actuator Dynamics for Active Structural Control," Joumal of Intelligent Materials, Systems, and Structures. vol. 1, pp. 327-354, July 1990.

[7] N. Hogan, "Impedance Control: An Approach to Manipulation," ASME Joumal of Dynamic Systems, Measurement, and Control, vol. 107, pp. 1-24, 1985.

[8] M. Jouaneh and H. Tian, "Accuracy Enhancement of a Piezoelectric Actuntor with Hysteresis," ASME JAPAN/USA Symposium on Flexible Ausomation, vol. 1, pp. 31-637, 1992.

[9] B. J. Lazan. Damping of Materials and Members in Structural Mechanics. Pergamon Press, London, 1968.
||$(0 \mid 1$ 1: 1.ce. T.J. Mom, and G.Y. Masidda, "Extended Bond Graph Reticulatinn of Picroclectric Continua.." ASME Jonernal of Dynamic Syisems, Measwement. and Control, vol. 117, no. 1. March. pp. 1-7, 1995.

|11|TD. Leigh and D.C. Zimmermau,. "An Implicit Method for the Nonlinaar Moklelling and Sinulation of Piezoceramic Actuators Displaying Hysleresis." ASNE Smart Siructures and Materials. AD-vol. 24. pp. 57-63. (1)i)

II:| C. Liang. F. Sun, and C.A. Rogers, "Coupled Electro-Mechanical Analysis of Piezoelectric Ceramic Actuator-Driven Systems: Determination wh the Actuator Power Consumption and System Energy Transfer," Proceeding. of the SPIE Smart Siructures and Intelligent Systems. vol. 1917, pp. 2.86-2019. 1993.

[13] M. Raiber and J. Craig, "Hybrid Position/Force Conirol of Manipulators." ASME Journal of Dynamic Systems, Measurement, and Control, vol. 103, pp. 126-133, 1981.

$|1+|$ H. Schempt and D.R. Yoerger, "Study of Dominant Performance Characteristics in Robot Transmissions." ASME Joumal of Mechanical Design. vol. 115 num. 3, pp. 472-482, 1993.

[15] Standards Committee of the LEEE Ultrasonics, Ferroelectrics, and Frequency Control Society, An American National Standand: IEEE Standard on Piezoelectriciry. The Institute of Electrical and Electronics Engineers, ANSUIEEE Sid. 176-1987, New York. 1987.

[16] W. Townsend and J.K. Salisbury, "The Effect of Coulomb Friction and Stiction on Force Control," in Proceedings of the IEEE Conference on Robotics and Auromation, pp. 883-889, April 1987.

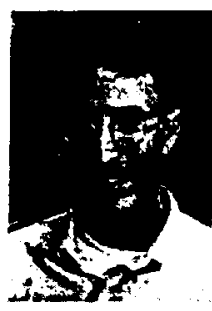

Michael Goldfarb received the B.S. degree in mechanical engineering from the University of Arizona in 1988, and the S.M. and Ph.D. degrees in mechanical engineering from the Massachusetts Institute of Technology in 1992 and 1994, respectively. He is currently Assistant Professor of Mechanical Engineering at Vanderbilt University. His research interests include design, modeling, and control of electromechanical systems, development of microrobotic and telerobotic systems, hybrid position/force control of robotic manipulators, and simulated mechanical environments.

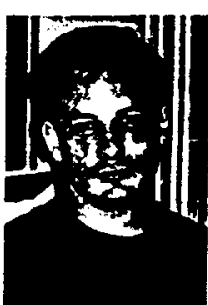

Nikola Celanovic received the B.S. degree in industrial electronics and telecommunications from the Univer: sity of Novi Sad in 1994 and the M.S. degree in meechanical engineering from Vanderbilt University in 1996. His research interests include robotics, control theory, and power electronics. He is currently pursuing a doctoral degree in power electronics at Virginia Polytechnic University and State School. 